\title{
recillunds
}

Revista Científica Mundo de la Investigación y el Conocimiento

Mónica Alexandra Aragadbay Torres ${ }^{a}$; Nervo Medrano Núñez ${ }^{b}$

Nivel de conocimiento de los estudiantes de 10mo semestre de la Facultad Piloto de

Odontología de la Universidad de Guayaquil sobre el manejo clínico de dientes permanentes avulsionados en niños de etapa escolar

Level of knowledge of the 10th semester students of the Pilot School of Dentistry of the University of Guayaquil on the clinical management of avulsed permanent teeth in school children

Revista Científica Mundo de la Investigación y el Conocimiento. Vol. 3 núm.3. Esp., noviembre, ISSN: 2588-073X, 2019, pp. 770-792

DOI: 10.26820/recimundo/3.(3.Esp).noviembre.2019.770-792

URL: $\underline{\text { htp://recimundo.com/index.php/es/article/view/626 }}$

Código UNESCO: 3213.13 Estomatología

Tipo de Investigación: Artículo de Revisión

(C) RECIMUNDO; Editorial Saberes del Conocimiento, 2019

Recibido: 15/09/2019

Aceptado: 23/10/2019

Publicado: 30/11/2019

Correspondencia: nervo.medranon@ug.edu.ec

a. Odontóloga; Investigadora Independiente; Guayaquil, Ecuador.

b. Diplomado en Docencia Superior; Especialista en Implantología Buco Maxilo Facial; Doctor en Odontología; Universidad de Guayaquil; Guayaquil, Ecuador; nervo.medranon@ug.edu.ec 


\section{Nivel de conocimiento de los estudiantes de 10mo semestre de la Facultad piloto de Odontología de la Universidad de Guayaquil sobre el manejo clínico de dientes permanentes avulsionados en niños de etapa escolar Vol. 3, núm. 3 Esp., (2019) \\ Mónica Alexandra Aragadbay Torres; Nervo Medrano Núñez}

\section{RESUMEN}

Los traumatismos dentales por avulsión son eventos dramáticos que usualmente se manifiestan en niños, aunque su incidencia sea baja del $1-6 \%$ en dentición permanente se considera uno de los traumatismos dentales más serios cuyo pronóstico depende de que tan rápido se asista. El desinterés de aprender de los estudiantes me motiva a averiguar qué tan preparados académicamente se encuentran. Siendo el objetivo de este estudio determinar el nivel de conocimiento de los estudiantes de 10mo semestre de la Facultad Piloto de Odontología de la Universidad de Guayaquil, sobre el manejo clínico de dientes permanentes avulsionados en niños de edad escolar. Consecuentemente se realizó un estudio de tipo científico en una muestra de 154 estudiantes, participando en una encuesta de 14 preguntas objetivas basadas en criterios de avulsión dentaria y tratamiento cuyos resultados son descritos en porcentajes representados en datos estadísticos y finalmente extrapolados a una valoración de nivel de conocimiento alto, medio o bajo. Los hallazgos sugieren que el 5\% de los estudiantes corresponde a un nivel de conocimiento alto, el $51 \%$ a un nivel medio y el $44 \%$ a un nivel bajo. Concluyendo así, el $56 \%$ de los estudiantes obtuvieron resultados aceptables; mientras que un $44 \%$ obtuvieron resultados inaceptables; de esta manera se obtiene una valoración general de nivel de conocimiento medio en los estudiantes.

Palabras clave: Conocimiento de los estudiantes; Traumatismo; Avulsión; Tratamiento. 


\title{
Nivel de conocimiento de los estudiantes de 10mo semestre de la Facultad piloto de Odontología de la Universidad de Guayaquil sobre el manejo clínico de dientes permanentes avulsionados en niños de etapa escolar
}

Vol. 3, núm. 3 Esp., (2019)

Mónica Alexandra Aragadbay Torres; Nervo Medrano Núñez

\begin{abstract}
Dental trauma by avulsion are dramatic events that usually manifested in children. Although its incidence is low in range from 1 to $16 \%$ in permanent dentition is considered one of the most serious dental trauma whose prognosis depends on how quickly is assisted. The lack of interest in learning from students motivates me to find out how academically prepared they are. The ain of this study is to determine the level of knowledge of 10th-semester students of the dentistry faculty of the University of Guayaquil about the clinical management of avulsed permanent teeth in school-age children. Consequently, a scientific study was conducted on a sample of 154 students, participating in a survey of 14 objective questions based on criteria of dental avulsion and treatment whose results are described in percentages represented in statistical data and finally extrapolated to a high, medium or low level of knowledge. Findings suggested that $5 \%$ of the students correspond to a high level of knowledge, $51 \%$ to a medium level and $44 \%$ to a low level. In conclusion, $56 \%$ of the students obtained acceptable results; while $44 \%$ obtained unacceptable results; thus, obtaining a general assessment of the average level of knowledge in the students.
\end{abstract}

Key words: Student knowledge; Trauma; Avulsion; Treatment. 


\section{Nivel de conocimiento de los estudiantes de 10mo semestre de la Facultad piloto de Odontología de la Universidad de Guayaquil sobre el manejo clínico de dientes permanentes avulsionados en niños de etapa escolar Vol. 3, núm. 3 Esp., (2019) \\ Mónica Alexandra Aragadbay Torres; Nervo Medrano Núñez}

\section{Introducción.}

Los traumatismos dentales por avulsión son eventos dramáticos para quien lo padezca, por lo general, lo sufren niños en edad escolar, por la hiperactividad en que ellos se desenvuelven. Jens o. Andreasen, mostró que el $22 \%$ de niños han sufrido daños dentales traumáticos en dentición permanente, siendo la edad más propensa entre los 8 - 10 años de edad, en un estudio realizado en Dinamarca (Koch \& Odontopediatría, 2011, pág. 264).

Con respecto a los reportes de incidencia de dientes avulsionados se encuentran en rangos de $1-16 \%$ en la dentición permanente, con una alta prevalencia en varones. No deja de ser uno de los traumatismos dentales más serios donde llega a ocasionar una serie de problemas al niño, tantos físicos como psicológicos.

El tratamiento ideal de la avulsión es la Reimplantación donde cuyo pronóstico dependerá de la prontitud que es atendido. Una perdida dental afecta no tan sólo al diente avulsionado sino también funciones masticatorias, problemas de crecimiento y desarrollo maxilar, fonación y estética, sin dejar atrás la autoestima que es un factor muy importante en la vida del niño para poder desenvolverse en su entorno natural.

Es importante poder lidiar con este tipo de urgencias en nuestra consulta general, saber actuar con precisión y rapidez, para evitar consecuencias permanentes a lo largo de la vida del paciente. Por lo consiguiente es necesario poseer conocimiento acerca el tratamiento clínico de trauma dental por avulsión y que mejor que valorarlo a estudiantes que están próximos a 


\section{Nivel de conocimiento de los estudiantes de 10mo semestre de la Facultad piloto de Odontología de la Universidad de Guayaquil sobre el manejo clínico de dientes permanentes avulsionados en niños de etapa escolar}

Vol. 3, núm. 3 Esp., (2019)

Mónica Alexandra Aragadbay Torres; Nervo Medrano Núñez

culminar con su carrera Odontológica previo a la obtención de título como Odontólogos Generales.

En una avulsión dental existe trauma no tan solo del diente sino también de los tejidos blandos y duros que lo rodean, donde, además ocurre una serie de sucesos traumáticos tantos físicos como emocionales para el niño y/o padres, familiares, profesores, etc. Aunque su frecuencia sea baja del 1-16\% en dentición permanente, se considera uno de los traumatismos dentales más serios, cuyo pronóstico depende de la prontitud que es atendido, por lo general, este fenómeno afecta más a niños a de edad escolar 6-12 años porque es ahí donde existe el mayor riesgo de acontecimientos traumáticos debido a la hiperactividad que desarrollan en dicho momento de su vida.

Por tal razón es importante tanto para el odontólogo general como para el estudiante de Odontología en formación, poseer un conocimiento pertinente de acuerdo al manejo clínico de urgencia en dientes avulsionados, para tener un correcto tratamiento que sea preciso y eficaz para evitar consecuencias desfavorables permanentes en el paciente.

\section{Trauma dental por avulsión}

Una avulsión dental puede ocurrir en cualquier etapa de la vida, pero se relaciona principalmente en la edad escolar por la interacción e hiperactividad que el niño desarrolla en aquel momento de su vida. Los dientes que más se ven afectado en este hecho son los incisivos centrales superiores y el rango de edad más afectado por lo general es de, entre 8 a 10 años con una predisposición en el sexo masculino (Montoya, Mariño, \& Rojas, 2012). 


\section{Nivel de conocimiento de los estudiantes de 10mo semestre de la Facultad piloto de Odontología de la Universidad de Guayaquil sobre el manejo clínico de dientes permanentes avulsionados en niños de etapa escolar Vol. 3, núm. 3 Esp., (2019) \\ Mónica Alexandra Aragadbay Torres; Nervo Medrano Núñez}

Epidemiología

En 1986 Gutiérrez y colaboradores, presentaron las estadísticas de trauma del hospital general de Medellín y estos datos muestran una incidencia de 55.9\% para niños y $44.1 \%$ para niñas (Jaramillo, 2009, pág. 228).

Un estudio en Dinamarca Jens o. Andreasen, mostró que el $22 \%$ de los niños ha sufrido daños dentales traumáticos en la dentición permanente, siendo la edad más propensa para sufrir accidentes entre los 8 y 10 años de edad. Donde la lesión dental normalmente es en uno o dos de los dientes anteriores, siendo especialmente el incisivo central del maxilar superior más afectado. (Koch \& Poulsen, 2011, pág. 264).

Los reportes de incidencia de dientes avulsionados se encuentran en rangos de 1-16\% en la dentición permanente, con una alta prevalencia en varones, mientras, en dentición decidua el porcentaje varía entre $7-21 \%$. Afecta principalmente a incisivos centrales superiores y muy raramente a los inferiores y por lo general, está comprometido un solo diente (Oyanguren, 2011, pág. 28).

\section{Etiología}

Un trauma dental por avulsión puede verse en cualquier etapa de la vida de una persona, podría presentarse mientras los primeros años de vida, efectuado por caídas como efecto de la falta de coordinación al momento del aprendizaje al caminar. Luego, más tarde al ingresar a la escuela vuelve a reaparecer este fenómeno debido a los juegos, deportes e interacción que llevan con sus compañeros o incluso violencia entre sus compañeros y finalmente en la edad adulta 


\section{Nivel de conocimiento de los estudiantes de 10mo semestre de la Facultad piloto de Odontología de la Universidad de Guayaquil sobre el manejo clínico de dientes permanentes avulsionados en niños de etapa escolar}

Vol. 3, núm. 3 Esp., (2019)

Mónica Alexandra Aragadbay Torres; Nervo Medrano Núñez

podría llegar a verse la avulsión, aunque en un rango de menor incidencia (De la Teja-Ángeles, Villegas-García, Rodríguez-Ramírez, \& Durán-Gutiérrez, 2016).

Las caídas son la causa más frecuente en trauma dental por avulsión en niños, Bhar en 1990, demostró que, en niños de 6 años o mayores a esta edad, el $60 \%$ ha sufrido accidentes en el juego, en la práctica de deportes, en el manejo de bicicletas o cualquier otro vehículo de ruedas (patines, patinetas, etc.) (Bordoni, Escobar, \& Castillo, 2010, pág. 512).

- Otros factores relacionados a trauma dental por avulsión son los siguientes:

- Accidentes automovilísticos

- Niños con padecimientos compulsivos o epilépticos.

- Niños con incisivos protruidos, maloclusiones en clase II

- Maltrato infantil

- Accidentes por el uso de la bicicleta

- Práctica de deportes de contactos, donde la prevalencia llega a ser hasta en un 45\% (Zaldivar, 2017).

- Peleas y violencia, en niños de 9 -12 años se refleja causa más frecuente de lesiones dentales (luxaciones laterales, avulsión), causando una frecuencia del 42.5\% (Leyva Labrador, y otros, 2017, pág. 6).

Tabla 1: Cuadro representativo de Diagnóstico y situación clínica de dientes permanentes avulsionados con ápice cerrado y abierto 


\section{Nivel de conocimiento de los estudiantes de 10mo semestre de la Facultad piloto de Odontología de la Universidad de Guayaquil sobre el manejo clínico de dientes permanentes avulsionados en niños de etapa escolar Vol. 3, núm. 3 Esp., (2019) Mónica Alexandra Aragadbay Torres; Nervo Medrano Núñez}

\begin{tabular}{|c|c|}
\hline \multicolumn{2}{|c|}{ DIENTES PERMANENTES A VULSIONADOS } \\
\hline Ápice cerrado & Ápice abierto \\
\hline Diente reimplantado en el momento & Diente reimplantado en el momento \\
\hline $\begin{array}{c}\text { Dientes que se han mantuvo en solución } \\
\text { de almacenamiento o seco en un tiempo } \\
<\text { a } 60 \text { min. }\end{array}$ & $\begin{array}{c}\text { Dientes que se mantuvo en solución de } \\
\text { almacenamiento o seco en un tiempo < } \\
\text { a } 60 \text { min. }\end{array}$ \\
\hline $\begin{array}{c}\text { Diente mantenido seco por más de } 60 \\
\text { minutos. }\end{array}$ & Diente mantenido seco por más de 60 \\
minutos.
\end{tabular}

Fuente: Mónica Aragadbay Torres

Avulsión de dientes permanentes

De las lesiones traumáticas, las avulsiones dentarias forman parte de las lesiones dentales más graves y tienen un pronóstico más desfavorable, sin embargo, su prevalencia estadísticamente no es altamente significativa. Este traumatismo se denomina también exarticulación y se define como el desplazamiento absoluto del diente de su alvéolo, con la consecuente lesión de las fibras del ligamento periodontal y del paquete vasculonervioso (Céspedes, Jimenez, \& Ramírez, 2016, pág. 2).

Una vez diagnosticado la Avulsión dental, clínica y radiográficamente, lo más indicado en dicho momento es la reimplantación. El procedimiento para reimplantar dientes se ha descrito ya repetidamente, las directrices se basan en las investigaciones en animales y en experiencias clínicas con humanos. No obstante, aun siguiendo estas reglas, es imposible pronosticar el éxito de una reimplantación a largo plazo (WAES \& Stockli, 2002, pág. 355).

Tabla 2. Cuadro representativo del Tratamiento de Dientes Avulsionados con Ápice cerrado. 


\section{Nivel de conocimiento de los estudiantes de 10mo semestre de la Facultad piloto de Odontología de la Universidad de Guayaquil sobre el manejo clínico de dientes permanentes avulsionados en niños de etapa escolar}

Vol. 3, núm. 3 Esp., (2019)

Mónica Alexandra Aragadbay Torres; Nervo Medrano Núñez

\section{Tratamiento de Dientes Avulsionados con Ápice Cerrado}

Diagnóstico y situación clínica

\begin{tabular}{|c|c|c|c|}
\hline & $\begin{array}{c}\text { Diente reimplantado antes } \\
\text { de llegar a consulta. }\end{array}$ & $\begin{array}{l}\text { Diente que se mantuvo en } \\
\text { medio de almacenamiento } \\
\text { o seco durante un tiempo } \\
\text { menor a } 60 \text { min. }\end{array}$ & $\begin{array}{c}\text { Diente que ha estado } \\
\text { extraoralmente en un } \\
\text { medio seco por más de } 60 \\
\text { min. }\end{array}$ \\
\hline & 1. No extraer el diente. & 1.Anestesia local. & 1.sujetar el diente por la \\
\hline $\begin{array}{l}\text { Tratamiento } \\
\text { inmediato }\end{array}$ & $\begin{array}{l}\text { 2.Limpiar zona afectada con } \\
\text { agua, suero fisiológico o } \\
\text { clorhexidina } 0.12 \% \text {. } \\
\text { 3.Limpieza y sutura de tejidos } \\
\text { blando adyacentes (sólo si es } \\
\text { necesario). } \\
\text { 4.Verificación clínica y } \\
\text { radiográfica de la posición del } \\
\text { diente ya reimplantado. } \\
\text { 5.Ferulizar (1-2 semanas) } \\
\text { 6.Administración } \\
\text { dantibióticos } \\
\text { 7.Verificar si tiene vacuna } \\
\text { antitetánica. } \\
\text { 8.Iniciar tratamiento } \\
\text { endodóntico } 7 \text { a } 10 \text { días post } \\
\text { reimplante. }\end{array}$ & 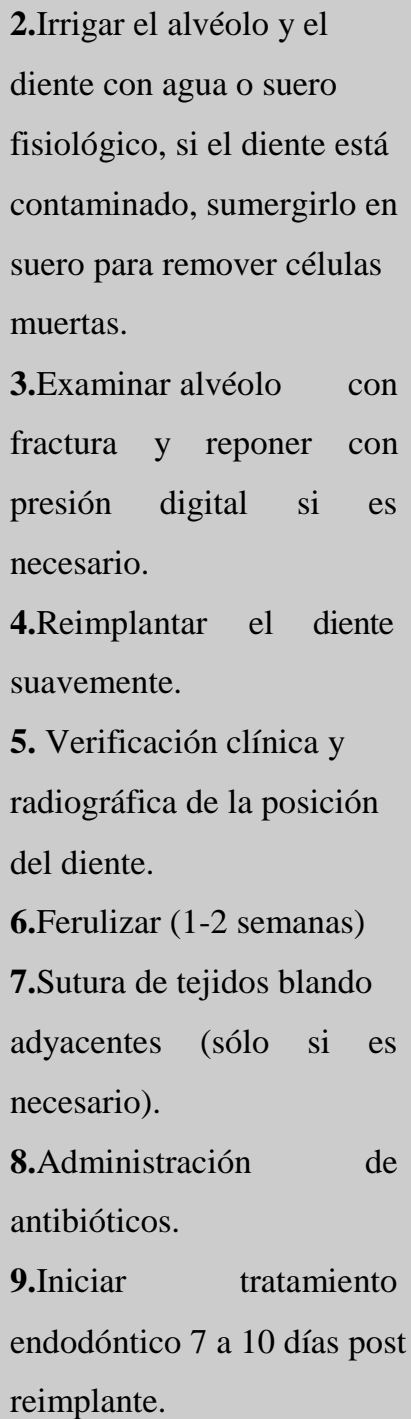 & 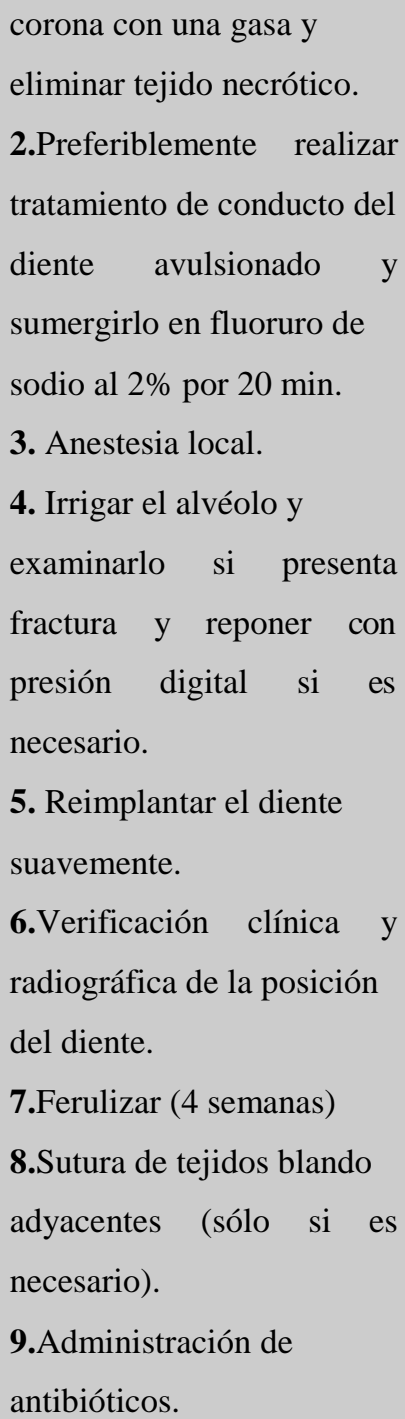 \\
\hline
\end{tabular}

Fuente: Mónica Aragadbay Torres. 


\section{Nivel de conocimiento de los estudiantes de 10mo semestre de la Facultad piloto de Odontología de la Universidad de Guayaquil sobre el manejo clínico de dientes permanentes avulsionados en niños de etapa escolar Vol. 3, núm. 3 Esp., (2019) \\ Mónica Alexandra Aragadbay Torres; Nervo Medrano Núñez}

Tabla 3: Cuadro representativo del Tratamiento de Dientes Avulsionados con Ápice Abierto

\begin{tabular}{|c|c|c|c|}
\hline \multicolumn{4}{|c|}{ Diagnóstico y situación clínica } \\
\hline & $\begin{array}{c}\text { Diente reimplantado } \\
\text { antes de llegar a } \\
\text { consulta. }\end{array}$ & $\begin{array}{l}\text { Diente que se mantuvo en } \\
\text { un medio de } \\
\text { almacenamiento o seco } \\
\text { durante un tiempo menor } \\
\text { a } 60 \mathrm{~min} .\end{array}$ & $\begin{array}{l}\text { Diente que ha estado } \\
\text { extraoralmente en un } \\
\text { medio seco por más de } \\
60 \text { min. }\end{array}$ \\
\hline $\begin{array}{l}\text { Tratamiento } \\
\text { inmediato }\end{array}$ & $\begin{array}{l}\text { 1.No extraer el diente. } \\
\text { 2.Limpiar zona afectada } \\
\text { con agua, suero fisiológico } \\
\text { o clorhexidina } 0.12 \% \text {. } \\
\text { 3.Limpieza y sutura de } \\
\text { tejidos blando adyacentes } \\
\text { (sólo si es necesario). } \\
\text { 4.Verificación clínica y } \\
\text { radiográfica de la posición } \\
\text { del diente ya } \\
\text { reimplantado. } \\
\text { 5.Ferulizar (1-2 semanas) } \\
\text { 6.Administración de } \\
\text { antibióticos } \\
\text { 7.verificar si tiene vacuna } \\
\text { antitetánica. } \\
\text { 8. Iniciar tratamiento } \\
\text { endodóntico } 7 \text { a } 10 \text { días } \\
\text { post reimplante. }\end{array}$ & $\begin{array}{l}\text { 1.Anestesia local. } \\
\text { 2.Irrigar el diente con } \\
\text { solución salina, sumergirlo } \\
\text { en } 20 \mathrm{ml} \text { de suero con } 1 \mathrm{mg} \text { de } \\
\text { dicloxacilina o minociclina } \\
\text { por } 5 \text { min. } \\
\text { 3.Irrigar y examinar el } \\
\text { alvéolo si presenta fractura y } \\
\text { reponer con presión digital si } \\
\text { es necesario. } \\
\text { 4.Reimplantar el diente } \\
\text { suavemente. } \\
\text { 5. Verificación clínica y } \\
\text { radiográfica de la posición } \\
\text { del diente. } \\
\text { 6.Ferulizar (1-2 semanas) } \\
\text { 7.Sutura de tejidos blando } \\
\text { Adyacentes (solo si es } \\
\text { necesario). } \\
\text { 8.Administración } \\
\text { antibióticos. } \\
\text { 9.Iniciar tratamiento } \\
\text { endodóntico } 7 \text { a } 10 \text { días post } \\
\text { reimplante. }\end{array}$ & $\begin{array}{l}\text { 1.sujetar el diente por la } \\
\text { corona con una gasa y } \\
\text { eliminar tejido necrótico. } \\
\text { 2.Preferiblemente realizar } \\
\text { el tratamiento de conducto } \\
\text { del diente avulsionado y } \\
\text { sumergirlo en fluoruro de } \\
\text { sodio al } 2 \% \text { por } 20 \text { min. } \\
\text { 3. Anestesia local. } \\
\text { 4. Irrigar el alvéolo y } \\
\text { examinarlo si presenta } \\
\text { fractura y reponer con } \\
\text { presión digital si es } \\
\text { necesario. } \\
\text { 5. Reimplantar el diente } \\
\text { suavemente. } \\
\text { 6.Verificación clínica y } \\
\text { radiográfica de la posición } \\
\text { del diente. } \\
\text { 7.Ferulizar (4 semanas) } \\
\text { 8.Sutura de tejidos blando } \\
\text { adyacentes (sólo si es } \\
\text { necesario). } \\
\text { 9.Administración } \\
\text { antibióticos. }\end{array}$ \\
\hline
\end{tabular}




\section{Nivel de conocimiento de los estudiantes de 10mo semestre de la Facultad piloto de Odontología de la Universidad de Guayaquil sobre el manejo clínico de dientes permanentes avulsionados en niños de etapa escolar \\ Vol. 3, núm. 3 Esp., (2019) \\ Mónica Alexandra Aragadbay Torres; Nervo Medrano Núñez}

Fuente: Mónica Aragadbay Torres.

Tabla 4. Cuadro representativo del control y seguimiento para dientes permanentes avulsionados con ápices abiertos y cerrados.

\begin{tabular}{|c|c|c|}
\hline Tiempo & $\begin{array}{l}\text { Tiempo extraoral inferior a } \\
\qquad 60 \mathrm{~min}\end{array}$ & $\begin{array}{l}\text { Ápice abierto sin importar } \\
\text { el tiempo extraoral }\end{array}$ \\
\hline 7-10 días & $\begin{array}{l}\text { Realizar el tratamiento de conducto, aplicación de Ca } \\
(\mathrm{OH}) \text {. }\end{array}$ & $\begin{array}{l}\text { No realizar tratamiento } \\
\text { endodóntico hasta q se } \\
\text { registren signos clínicos y } \\
\text { radiográficos de necrosis } \\
\text { pulpar }\end{array}$ \\
\hline 2 semanas & Extracción de Férula y Examen Radiológico & \\
\hline 4 semanas & $\begin{array}{l}\text { Examen clínico y radiológico y tratamiento } \\
\text { endodóntico definitivo }\end{array}$ & Examen clínico y radiológico. \\
\hline $\begin{array}{c}\text { 3-6-12 meses } \\
\text { y cada año } \\
\text { por cinco } \\
\text { años }\end{array}$ & Examen clínico y radiológico & Examen clínico yradiológico \\
\hline
\end{tabular}

Fuente: Mónica Aragadbay Torres.

\section{Metodología.}

Diseño y tipo de investigación

El diseño de la presente investigación es de tipo:

Cualicuantitativa: Porque se estableció un cuestionario de preguntas a los estudiantes de

10mo semestre del ciclo CII periodo 2018-2019 de la facultad piloto de Odontología de la 


\section{Nivel de conocimiento de los estudiantes de 10mo semestre de la Facultad piloto de Odontología de la Universidad de Guayaquil sobre el manejo clínico de dientes permanentes avulsionados en niños de etapa escolar \\ Vol. 3, núm. 3 Esp., (2019) \\ Mónica Alexandra Aragadbay Torres; Nervo Medrano Núñez}

Universidad de Guayaquil, para determinar el nivel de conocimiento acerca la avulsión dentaria permanente, la cual servirá para la recopilación de información cuantificables para la presentación de datos estadísticos que será convertida en una valoración cualitativa de nivel de conocimiento alto, medio o bajo.

No Experimental: Porque se va a identificar el nivel de conocimiento de los estudiantes a través de un cuestionario acerca avulsión dentaria permanente. Sin manejo de variables.

Descriptiva: con los datos obtenidos de la encuesta se presenta el nivel de conocimiento de los estudiantes, a través de una valoración propia del proyecto.

De campo: porque se la realizó a los estudiantes de 10mo semestre de la Facultad de Odontología dentro de las instalaciones de la Universidad de Guayaquil.

Documental: porque toda la información descrita en este trabajo es basada en guías prácticas de trauma dental, libros y artículos ya establecidos a nivel internacional.

Población y muestra

La población de la presente investigación está constituida por 250 estudiantes matriculados en 10mo semestre CII periodo 2018-2019 de la Facultad Piloto de Odontología de la Universidad de Guayaquil, según la base de datos de secretaría de esta Institución. 


\section{Nivel de conocimiento de los estudiantes de 10mo semestre de la Facultad piloto de Odontología de la Universidad de Guayaquil sobre el manejo clínico de dientes permanentes avulsionados en niños de etapa escolar}

Vol. 3, núm. 3 Esp., (2019)

Mónica Alexandra Aragadbay Torres; Nervo Medrano Núñez

El tamaño de la muestra de esta investigación está constituido por 154 estudiantes de 10mo semestre de la Facultad Piloto de Odontología de la Universidad de Guayaquil, del ciclo CII periodo 2018-2019.

\section{Técnicas e instrumentos}

- La técnica empleada en esta investigación es la encuesta direccionada a los estudiantes de décimo semestre que estuvieron de acuerdo en participar, se la realizó a 154 estudiantes, que pertenecen a jornada matutina y vespertina, los cuales se les realizó 14 preguntas de tipo objetivo, basadas en guías de manejo de trauma dental ya establecidas internacionalmente. La encuesta estuvo diseñada para contestarla personalmente, teniendo una sola respuesta en cada pregunta.

- El instrumento de recolección de información como ya se mencionó son las hojas de encuesta ya respondidas por los estudiantes, formulada con 14 preguntas y con una sola respuesta en cada una de ellas, divididas en cinco partes:

1. conocimientos de definición de avulsión,

2. tiempo y medio de almacenamiento ideal del diente permanente avulsionado hasta antes de su reimplante.

3. manejo clínico de dientes permanentes avulsionados,

4. procedimientos endodónticos posterior al reimplante

5. recomendaciones prescritas para la recuperación del paciente luego de la consulta odontológica. 


\section{Nivel de conocimiento de los estudiantes de 10mo semestre de la Facultad piloto de Odontología de la Universidad de Guayaquil sobre el manejo clínico de dientes permanentes avulsionados en niños de etapa escolar \\ Vol. 3, núm. 3 Esp., (2019) \\ Mónica Alexandra Aragadbay Torres; Nervo Medrano Núñez}

Cada una de ellas consta de una calificación de 0.72 puntos, donde, cuyo resultado se lo calcula por medio de porcentajes, se lo representa por cuadros estadísticos y se lo extrapola a una valoración de Nivel de conocimiento alto, medio o bajo. A continuación, la escala de valores:

Tabla 5. Cuadro representativo de la tabla de Valoración de Conocimiento.

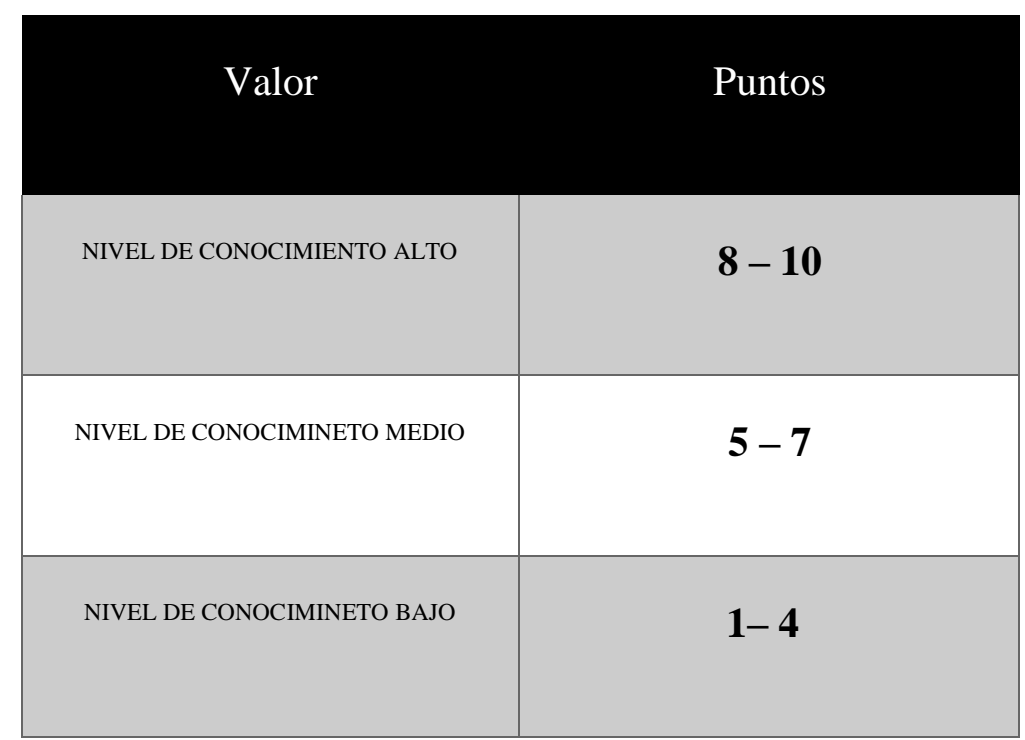

Fuente: Mónica Aragadbay Torres.

\section{Resultados.}

Del total de 154 estudiantes de 10mo semestre de muestra, se logra obtener el siguiente resultado: 147 estudiantes equivalente al 95\% de la muestra, identifican el término trauma dental por avulsión, mientras que el $4 \%$ de la muestra tiene un concepto equivocado del tema y el $1 \%$ definitivamente lo desconoce.

Se determina que 90 estudiantes correspondientes al $60 \%$ de la muestra, poseen conocimientos acertados acerca el tiempo ideal para el reimplante del diente avulsionado, 


\section{Nivel de conocimiento de los estudiantes de 10mo semestre de la Facultad piloto de Odontología de la Universidad de Guayaquil sobre el manejo clínico de dientes permanentes avulsionados en niños de etapa escolar}

Vol. 3, núm. 3 Esp., (2019)

Mónica Alexandra Aragadbay Torres; Nervo Medrano Núñez

mientras, que el 53 estudiante (34\%) poseen conocimientos equivocados y 9 estudiantes (6\%) totalmente desconocen del tema.

Se determina que 49 estudiantes correspondiente al 32\% de la muestra, reconocen la solución salina balanceada de Hank como medio ideal de almacenamiento del diente avulsionado, mientras, que el 97 estudiante (63\%) poseen conocimientos equivocados y 8 estudiantes $(5 \%)$ totalmente desconocen del tema.

Se estableció que 81 estudiantes que equivale al 53\% de la muestra, saben que se debe realizar una limpieza en el alveolo una vez dentro del consultorio, 64 estudiante (42\%) poseen conocimientos equivocados y 9 estudiantes (5\%) totalmente desconocen del tema.

Se establece que 111 estudiantes que equivale al (72\%) de la muestra, conocen que no se debe extraer un diente ya reimplantado antes de llegar a la consulta, 32 estudiante (21\%) poseen conocimientos equivocados y 11 estudiantes $(7 \%)$ totalmente desconocen del tema.

Con respecto a esta pregunta, se establece que solo 23 estudiantes lo que equivale al (15\%) de la muestra, conocen que el tiempo que debe permanecer la férula en el diente reimplantado es de 1-2 semanas, 111 estudiante (72\%) poseen conocimientos equivocados y 20 estudiantes (13\%) totalmente desconocen del tema.

Con respecto a esta pregunta, se establece que 76 estudiantes lo que equivale al (49\%) de la muestra, indicaron que del tipo de férula es la ideal para ferulizar un diente avulsionado es la semirrígida, 61 estudiante (40\%) poseen conocimientos erróneos y 17 estudiantes (11\%) totalmente desconocen del tema. 


\section{Nivel de conocimiento de los estudiantes de 10mo semestre de la Facultad piloto de Odontología de la Universidad de Guayaquil sobre el manejo clínico de dientes permanentes avulsionados en niños de etapa escolar Vol. 3, núm. 3 Esp., (2019) Mónica Alexandra Aragadbay Torres; Nervo Medrano Núñez}

Con respecto a esta pregunta, se puede identificar que solo 35 estudiantes lo que equivale al (23\%) de la muestra, indicaron que en estos casos el tratamiento de conducto se debe realizar entre 7-10 días aproximadamente posterior al reimplante, 71 estudiante (46\%) poseen conocimientos erróneos y 48 estudiantes (31\%) totalmente desconocen del tema.

Se puede identificar que 48 estudiantes lo que equivale al (31\%) de la muestra, indicaron que en estos casos el tratamiento de conducto se debe realizar entre inmediatamente antes de reimplante, 52 estudiante (34\%) poseen conocimientos erróneos y 54 estudiantes (35\%) totalmente desconocen del tema.

Se puede identificar que 80 estudiantes lo que equivale al (52\%) de la muestra, indicaron que en estos casos el tratamiento de conducto debe evitarse hasta presenciar hallazgos desfavorables tantos clínicos como radiológicos, 38 estudiante (24\%) poseen conocimientos erróneos y 36 estudiantes (24\%) totalmente desconocen del tema.

Se puede identificar que 120 estudiantes lo que equivale al (78\%) de la muestra, indican que el Hidróxido de calcio es la medicación más recomendable para tratamiento de conductos de dientes avulsionados, 21 estudiante (13\%) poseen conocimientos erróneos y 13 estudiantes (9\%) totalmente desconocen del tema.

Con respecto a esta pregunta, se puede determinar que solo 28 estudiantes lo que equivale al (18\%) de la muestra, poseen conocimientos acertados acerca de las recomendaciones y cuidados en casa para el paciente, 113 estudiante (74\%) poseen conocimientos erróneos y 13 estudiantes (8\%) totalmente desconocen del tema. 


\section{Nivel de conocimiento de los estudiantes de 10mo semestre de la Facultad piloto de Odontología de la Universidad de Guayaquil sobre el manejo clínico de dientes permanentes avulsionados en niños de etapa escolar}

Vol. 3, núm. 3 Esp., (2019)

Mónica Alexandra Aragadbay Torres; Nervo Medrano Núñez

Se determina que 96 estudiantes lo que equivale al (62\%) de la muestra, indicaron que se debe recomendar la aplicación de la vacuna antitetánica, 32 estudiante (21\%) poseen conocimientos erróneos y 26 estudiantes (17\%) totalmente desconocen del tema.

Con respecto a esta pregunta, se puede determinar que 48 estudiantes lo que equivale al (31\%) de la muestra, poseen conocimientos acertados acerca del seguimiento y control al paciente, 83 estudiante (54\%) poseen conocimientos erróneos y 23 estudiantes (15\%) totalmente desconocen del tema.

8 encuestas obtuvieron una calificación de nivel de conocimiento alto lo que corresponde al 5\% de la muestra encuestada, 78 encuestas obtuvieron una calificación de nivel de conocimiento medio lo que equivale al $51 \%$ de la muestra y por último 68 encuestas obtuvieron una calificación de nivel de conocimiento bajo lo que equivale al 44\% de la población.

Finalmente, el valor promedio general es de 4.83 que redondeándolo da un valor de 5 sobre 10 puntos lo que determina un nivel de conocimiento medio.

\section{Discusión}

En este estudio de nivel de conocimiento de estudiantes de 10mo semestre de la Facultad de Odontología realizado en la Universidad de Guayaquil, en forma general, podemos comprobar que los conocimientos acerca de trauma dental por avulsión son aceptable en un 56\% de la muestra de los estudiantes, así mismo el 44\% restante de la muestra de los estudiantes poseen un resultado totalmente desfavorable, mostrando resultados similares en los estudios realizados por Lora Salgado y colaboradores en la Universidad del Sinú Cartagena Colombia 


\section{Nivel de conocimiento de los estudiantes de 10mo semestre de la Facultad piloto de Odontología de la Universidad de Guayaquil sobre el manejo clínico de dientes permanentes avulsionados en niños de etapa escolar Vol. 3, núm. 3 Esp., (2019) Mónica Alexandra Aragadbay Torres; Nervo Medrano Núñez}

acerca de conocimientos, actitudes y experiencias respecto a traumatología dental, donde se determinó un nivel de conocimiento aceptable en un $64.4 \%$, actitudes desfavorables en un $82 \%$ y experiencias del $95 \%$ de los estudiantes que nunca se han cruzado con pacientes con traumas dentales por avulsión (Lora-Salgado, y otros, 2018, págs. 1-2).

Según el Primer objetivo específico de este estudio "Identificar si el estudiante conoce el termino trauma dental por avulsión“, podemos comprobar que un $95 \square$ de los estudiantes conocen el concepto de avulsión dental. Para el Segundo objetivo específico “Determinar si el estudiante conoce el tiempo y el medio de almacenamiento ideal del diente avulsionado“, se determina que el $60 \square$ de los estudiantes consideran el tiempo inferior a 15 minutos como tiempo ideal de reimplante del diente avulsionado para un mejor pronóstico, mientras que, con respecto a la solución ideal de almacenamiento o preservación de un dientes avulsionado, solo el 32\% de la muestra de los estudiantes seleccionó la solución salina balanceada de Hank.

Con respecto al Tercer objetivo específico "Establecer si el estudiante conoce el manejo clínico de dientes permanentes avulsionados" se lo logra obtener con cuatro preguntas en la encuesta, cuyos resultados son los siguientes: el 53\% de los encuestados aseguran que se debe realizar limpieza en el alveolo con agua, suero o clorhexidina al $0.12 \%$ en el consultorio antes de proceder con el tratamiento, afortunadamente el $72 \%$ indican que no se debe extraer un diente avulsionado que llega a consulta con el diente ya reimplantado, sin embargo, solo el $15 \%$ acertó con respecto al tiempo establecido de permanencia de la férula de 1-2 semanas en el diente avulsionado, pero, solo el $49 \%$ indicó que el tipo de férula más adecuada en este tipo de trauma dental es la Férula semirrígida, mostrando resultados similares en estudios realizados por 


\section{Nivel de conocimiento de los estudiantes de 10mo semestre de la Facultad piloto de Odontología de la Universidad de Guayaquil sobre el manejo clínico de dientes permanentes avulsionados en niños de etapa escolar}

Vol. 3, núm. 3 Esp., (2019)

Mónica Alexandra Aragadbay Torres; Nervo Medrano Núñez

Emien y Omole (2015) en Nigeria, donde se encuestó a estudiantes de medicina para evaluar el conocimiento y la práctica en manejo de emergencias del trauma dental, donde se determinó que un $83 \%$ de los estudiantes conocían el concepto de definición de avulsión dentaria, solo el 15\% sabía que el tratamiento ideal de la avulsión es la reimplantación, en cuanto al tiempo de reimplante determinaron que el $25.5 \%$ de los estudiantes conocen que el diente debe recolocarse dentro de los 0-60min y la mayoría 51.1\% seleccionó a la solución salina como medio para la preservación de un diente avulsionado (Joan Emien \& Omo Julie, 2015, pág. 54).

Para el Cuarto objetivo específico identificar acerca de los procedimientos endodónticos post al reimplante que conoce el estudiante se lo logra identificar por medio de cuatro preguntas en la encuesta, cuyos resultados son los siguientes: solo el $23 \%$ de los encuestados indicaron que el tratamiento de conducto de dientes permanentes con ápice cerrado se realiza a los 7-10 días. El $31 \%$ indicó que el tratamiento de conducto se realiza inmediatamente cuando el diente ha permanecido avulsionado por más de 60 minutos. El 52\% afirma que el tratamiento de conducto debe evitarse cuando el diente avulsionado presenta ápice abierto y se mantuvo húmedo en un tiempo inferior a 60 minutos. Y afortunadamente, un $78 \square$ de los encuestados afirma que el hidróxido de calcio es la medicación intraconducto más recomendada en casos de dientes con trauma por avulsión.

En casos de control y seguimiento lo que corresponde al quinto objetivo específico es determinar si el estudiante conoce acerca las recomendaciones prescritas para la recuperación de los pacientes luego de la consulta odontológica“ se lo logra determinar a partir de tres preguntas en la encuesta, cuyos resultados son los siguientes: solo el $18 \%$ de los encuestados 


\section{Nivel de conocimiento de los estudiantes de 10mo semestre de la Facultad piloto de Odontología de la Universidad de Guayaquil sobre el manejo clínico de dientes permanentes avulsionados en niños de etapa escolar Vol. 3, núm. 3 Esp., (2019) Mónica Alexandra Aragadbay Torres; Nervo Medrano Núñez}

conoce las recomendaciones de dieta, cuidados e higiene que este tipo de paciente requiere, la mayoría de los estudiantes lo que equivale el $62 \%$ indican que se debe recomendar la aplicación de la vacuna antitetánica, mientras que solo el $31 \%$ de estudiantes, poseen conocimientos acertados sobre el control y seguimiento que se le da al paciente, al igual, que en el estudio realizado por Valdepeñas Morales, J. y colaboradores (2016) en Madrid, donde se observa que el conocimiento sobre el manejo del trauma dental y el su control y seguimiento el $92.31 \%$ de los profesionales sanitarios especialistas en Odontología tenían nociones insuficientes (Valdepeñas Morales, Adanero Velasco, \& Planells del Pozo, 2016).

Con respecto al sexto objetivo específico "Valorar el nivel de conocimiento de los estudiantes", se lo pudo lograr a partir de los resultados de la encuesta, donde dio como calificación general 5 sobre 10 puntos. Por todo esto y lo anteriormente planteado se logar determinar que el nivel de conocimiento de los estudiantes de 10mo semestre de la facultad piloto de odontología de la universidad de Guayaquil toma un valor basado según la escala de valoración realizada por mi autoría, como una valoración de conocimientos medio, es decir, que se demuestra un déficit de conocimiento en áreas Odontológicas, acerca del manejo clínico del tema de traumatología dental por avulsión dentaria por parte de los estudiantes, como es el caso del estudio realizado por Valdepeñas Morales, J. y colaboradores (2016) realizados a profesionales sanitarios de Atención Primaria de la Comunidad de Madrid, acerca del manejo del traumatismo dental por avulsión los grupos presentaron conocimientos limitados, excepto el grupo de odontólogos, los cuales obtuvieron un resultado de competencia adecuada. Sin embargo, se encontró que el 92\% de los odontólogos tenían nociones insuficientes en relación a 


\section{Nivel de conocimiento de los estudiantes de 10mo semestre de la Facultad piloto de Odontología de la Universidad de Guayaquil sobre el manejo clínico de dientes permanentes avulsionados en niños de etapa escolar}

Vol. 3, núm. 3 Esp., (2019)

Mónica Alexandra Aragadbay Torres; Nervo Medrano Núñez

los protocolos actualizados sobre la atención en trauma dental por avulsión y control y seguimiento (Valdepeñas Morales, Adanero Velasco, \& Planells del Pozo, 2016, pág. 33).

\section{Conclusiones.}

- Se realizó una encuesta a 154 estudiantes de 10mo semestre sobre manejo clínico de dientes permanentes avulsionados en niños de edad escolar, para determinar su nivel de conocimiento.

- Obteniendo así un 5\% de la muestra de los estudiantes, una valoración de nivel conocimiento alto, un 51\% un nivel medio, y el $44 \square$ un nivel bajo.

- Lo que indica que un $56 \%$ se obtiene un resultado favorable mientras que un $44 \%$ un resultado desfavorable, con lo cual se puede determinar que el nivel de conocimiento de los estudiantes de 10mo semestre de la facultad piloto de odontología de la universidad de Guayaquil toma un valor basado según la escala de valoración realizada por mi autoría, como una valoración de conocimiento medio.

\section{Recomendaciones}

Se sugiere que, durante el periodo de formación de los estudiantes de la carrera de odontología, exista mayor énfasis con respecto a la enseña de los temas de traumatismos dentales por avulsión, siendo este un tema importante y de constante actualización. A su vez mayor interés por parte del estudiante para la lograr obtener mejores conocimientos acerca del tema y poder desempeñar mejor su labor. 


\section{Nivel de conocimiento de los estudiantes de 10mo semestre de la Facultad piloto de Odontología de la Universidad de Guayaquil sobre el manejo clínico de dientes permanentes avulsionados en niños de etapa escolar Vol. 3, núm. 3 Esp., (2019) Mónica Alexandra Aragadbay Torres; Nervo Medrano Núñez}

Existen protocolos y guías de manejo clínico de trauma dental, científicamente comprobados, de fácil acceso por vía internet y sobre todo de fácil compresión para reforzar conocimientos ya establecidos, los cuales pudieran utilizarse como medios de capacitación e ilustración de los estudiantes acerca del tema. Tomando en cuenta que dichos conocimientos deben se actualizados constantemente para poder asistir de una manera oportuna si llegase a presentarse este caso en la consulta odontológica.

Existen protocolos y guías de manejo clínico de trauma dental, científicamente comprobados, de fácil acceso por vía internet y sobre todo de fácil compresión para reforzar conocimientos ya establecidos, los cuales pudieran utilizarse como medios de capacitación e ilustración de los estudiantes acerca del tema.

\section{Bibliografía.}

Bordoni, N., Escobar, A., \& Castillo, R. (2010). Odontología Pediátrica La salud del niño y el adolescente en el mundo actual. Ira edición. Buenos Aires: Medica Panamericana .

Céspedes, L. I., Jimenez, M., \& Ramírez, Y. (2016). Exarticulación dentaria. Revista Electrónica Dr. Zoilo E. Marinello Vidaurreta, 41(3), 1-4.

De la Teja-Ángeles, E., Villegas-García, L., Rodríguez-Ramírez, F., \& Durán-Gutiérrez, L. (2016). Avulsión dental. Acta Pediátrica de México, 37(2), 132-133.

Jaramillo, D. (2009). Fundamentos de odontología. Odontología Pediátrica. Medellín, Colombia: OD, MSC Corporación para investigaciones Biológicas.

Joan Emien, E., \& Omo Julie, O. (06 de 2015). Knowledge and Attitudes of final year. Journal of Biomedical Sciences, 14(1), 54-63. Obtenido de https://www.ajol.info/index.php/jmbr/article/view/124878/114396

Koch, G., \& Poulsen, S. (2011). Odontopediatria. Abordaje clínico. 
Nivel de conocimiento de los estudiantes de 10mo semestre de la Facultad piloto de Odontología de la Universidad de Guayaquil sobre el manejo clínico de dientes permanentes avulsionados en niños de etapa escolar

Vol. 3, núm. 3 Esp., (2019)

Mónica Alexandra Aragadbay Torres; Nervo Medrano Núñez

Leyva Labrador, Y., Baile Labrador, Y., Ribeaux Chibás, A., Rodríguez Trejo, E., Suárez Zafra, D., Sánchez Fernández, N., \& Castellanos González, M. (08 de 2017). Revisión Bibliográfica sobre trauma dental en niños. Obtenido de Unirioja: https://dialnet.unirioja.es/descarga/articulo/6143847.pdf

Lora-Salgado, I. M., Tirado-Amador, L., Vargas-Quiroga, E., Barcha-Barreto, D., LombanaOrtiz, L, Lombana-Ortiz, L., . . . Ortiz-Brun, G. (2018). Conocimiento, actitudes y experiencia respecto a trauma dental en estudiantes de odontología en Cartagena Colombia. Revista Nacional de Odontología, 14(26), 1-11.

Montoya, J. H., Mariño, F., \& Rojas, H. (2012). Protocolo de manejo de diente permanente avulsionado para el servicio de salud oral de la Fundación Hospital La Misericordia y la Facultad de Odontología de la Universidad Nacional de Colombia, sede Bogotá (2011). Universitas Odontológica, 31(66), 185-210.

Oyanguren, S. (2011). Medios de almacenamiento para preservar dientes avulsionados. Revista Odontológica Pediátrica, 10(1), 517-531.

Valdepeñas Morales, J., Adanero Velasco, A., \& Planells del Pozo, P. (2016). Estado actual de los conocimientos sobre el manejo de los traumatismos dentales en los profesionales sanitarios de Atención Primaria de la Comunidad de Madrid. Estudio piloto. Cient. dent, 13(12), 113-122.

Waes, H. J., \& Stockli, P. W. (2002). Atlas de odontología pediátrica. Barcelona: Masson.

Zaldivar, H. A. (2017). Traumatismos dentarios: un acercamiento imprescindible. Rev 16 de Abril, 56(265), 113-118.

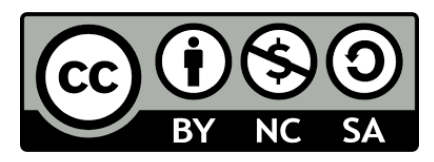

RECONOCIMIENTO-NOCOMERCIAL-COMPARTIRIGUAL

CC BY-NC-SA

ESTA LICENCIA PERMITE A OTROS ENTREMEZCLAR, AJUSTAR Y CONSTRUIR A PARTIR DE SU OBRA CON FINES NO COMERCIALES, SIEMPRE Y CUANDO LE RECONOZCAN LA AUTORÍA Y SUS NUEVAS CREACIONES ESTÉN BAJO UNA LICENCIA CON LOS MISMOS TÉRMINOS. 